\title{
Transmuted Lower Record Type Fréchet Distribution with Lifetime Regression Analysis Based on Type I-Censored Data
}

\author{
Caner Tanış ${ }^{1, \star}$, Buğra Saraçoğlu ${ }^{2}$, Coşkun Kuş ${ }^{2}$, Ahmet Pekgör ${ }^{3}$, Kadir Karakaya ${ }^{2}$ \\ ${ }^{1}$ Department of Statistics, Faculty of Science, Cankiri Karatekin University, Cankiri, 18100, Turkey \\ ${ }^{2}$ Department of Statistics, Faculty of Science, Selçuk University, Konya, 42250, Turkey \\ ${ }^{3}$ Department of Statistics, Faculty of Science, Necmettin Erbakan University, Konya, 42090, Turkey
}

\section{ARTICLE INFO}

Article History

Received 15 Apr 2019

Accepted 20 Nov 2020

Keywords

Fréchet distribution

Lower records

Point estimates

Lifetime regression

2000 Mathematics Subject

Classification: $60 \mathrm{E} 05,62 \mathrm{E} 15,62 \mathrm{~F} 10$

\begin{abstract}
This paper introduces a new lifetime distribution by mixing the first two lower record values and various distributional properties are examined. Statistical inference on distribution parameters are discussed with five estimators. A Monte Carlo simulation study is carried out to evaluate the risk behavior of these estimators for different sample of sizes. The distribution modeling analysis is provided based on real data to demonstrate the fitting ability of the proposed model. In addition, a lifetime regression model is described by re-parameterization on the log lifetimes. The superiority of proposed regression model is revealed in well-known
\end{abstract} models.

(C) 2021 The Authors. Published by Atlantis Press B.V. This is an open access article distributed under the CC BY-NC 4.0 license (http://creativecommons.org/licenses/by-nc/4.0/).

\section{INTRODUCTION}

In two decades, many statistical distributions have been brought into the literature. These distributions are obtained as a member of the family of distributions. Transmuted families can be given as an example to the family of distributions. In general, transmuted families are given based on order statistics. The transmuted distributions has been introduced by Shaw and Buckley [1,2] using a quadratic transmutation map. Order statistics, transmuted distributions can be sorted as [3-5]. Recently, Balakrishnan [6] proposed a new record based transmuted family of distributions. Tanış and Saraçoğlu [7] examined a special model based on Weibull distribution of record based transmuted family of distributions. In terms of both distributional properties and statistical inference, in parallel with the study of [6].

In our study, we obtained the transmuted version of the Fréchet distribution based on lower record values. In Section 2, a transmutation lower record type map of order 2 is introduced. In Section 3, we have suggested a sub-model called transmuted lower record type Fréchet (TLRTF) distribution and the some distributional properties are obtained such as moments, incomplete moments, Bonferroni and Lorenz curves. In Section 4, the unknown parameters are estimated by five methods including maximum likelihood estimators, least squares estimators, weighted least squares estimators, Anderson-Darling estimators, and Cramer von-Mises estimators. A simulation study is performed in order to compare the performance of these estimators in terms of mean squared errors (MSEs) and bias. A lifetime regression is introduced based on TLRT-F distribution in Section 5. In Sections 6 and 7, two applications with real data are presented to show the applicability of introduced distribution.

\section{TRANSMUTATION LOWER RECORD TYPE MAP OF ORDER 2}

Let $X_{L(1)}$ and $X_{L(2)}$ be the lower record values from a population with cumulative distribution function (cdf) $G(x)$. Let us define a new random variable based on these records:

$$
Y= \begin{cases}X_{L(1)}, & U<p \\ X_{L(2)}, & U>p,\end{cases}
$$


where $U$ is standard uniform random variable and $p \in(0,1)$. The cdf of $Y$ is written by

$$
\begin{aligned}
F(x) & =p P\left(X_{L(1)} \leq x\right)+(1-p) P\left(X_{L(2)} \leq x\right) \\
& =p G(x)+(1-p)[G(x)(1-\log (G(x)))] \\
& =G(x)[1-p \log (G(x))] .
\end{aligned}
$$

It is noticed that the distribution with cdf (2.2) is called "transmuted lower record type distribution (TLRT)." Using (2.2), the probability density function (pdf) and hazard function(hf) of TLRT distribution are given by

$$
f(x)=g(x)[1-p(1+\log (G(x)))],
$$

and

$$
h(x)=\frac{g(x)[1-p(1+\log (G(x)))]}{1-G(x)[1-p \log (G(x))]}
$$

respectively.

\section{TLRT-F DISTRIBUTION AND DISTRIBUTIONAL PROPERTIES}

In this section, we investigate the sub-model of TLRT family. Let $X$ be a random variable having Fréchet distribution. The cdf and pdf of $X$ are defined by

$$
G(x)=\exp \left(-\left(\frac{\beta}{x}\right)^{\alpha}\right)
$$

and

$$
g(x)=\alpha \beta^{\alpha} x^{-(\alpha+1)} \exp \left(-\left(\frac{\beta}{x}\right)^{\alpha}\right), x>0
$$

respectively, where $\alpha>0$ is a shape parameter and $\beta>0$ is scale parameter. Substituting the cdf (3.1) and pdf (3.2) into (2.2) and (2.3), the following cdf and pdf are obtained as

$$
F(x ; \theta)=\exp \left(-\left(\frac{\beta}{x}\right)^{\alpha}\right)\left(1+p\left(\frac{\beta}{x}\right)^{\alpha}\right)
$$

and

$$
f(x ; \theta)=\alpha \beta^{\alpha} x^{-(\alpha+1)} \exp \left(-\left(\frac{\beta}{x}\right)^{\alpha}\right)\left(1-p+p\left(\frac{\beta}{x}\right)^{\alpha}\right), x>0
$$

where $\alpha, \beta>0, p \in(0,1)$, and $\boldsymbol{\theta}=(\alpha, \beta, p)$. The distribution with cdf (3.3) is called TLRT-F ( $\boldsymbol{\theta})$ distribution.

The hf of TLRT-F distribution is given by

$$
h(x ; \theta)=\frac{\alpha \beta^{\alpha} x^{-(\alpha+1)} \exp \left(-\left(\frac{\beta}{x}\right)^{\alpha}\right)\left(1-p+p\left(\frac{\beta}{x}\right)^{\alpha}\right)}{1-\exp \left(-\left(\frac{\beta}{x}\right)^{\alpha}\right)\left(1+p\left(\frac{\beta}{x}\right)^{\alpha}\right)} .
$$

Figures 1-2 illustrates the possible shapes of pdf and hf for selected parameters. The quantile function of the TLRT-F is given by

$$
Q(q ; \boldsymbol{\theta})=\beta\left(-\frac{p W\left(\frac{q \exp (-1 / p)}{p}\right)+1}{p}\right)^{-\frac{1}{\alpha}},
$$

where $q \in[0,1]$ and $W(\cdot)$ is a Lambert function. Using (3.6), the median can be easily written by

$$
Q(0.5 ; \boldsymbol{\theta})=\beta\left[-\frac{p W\left(\frac{\exp (-1 / p)}{2 p}\right)+1}{p}\right]^{-\frac{1}{\alpha}}
$$



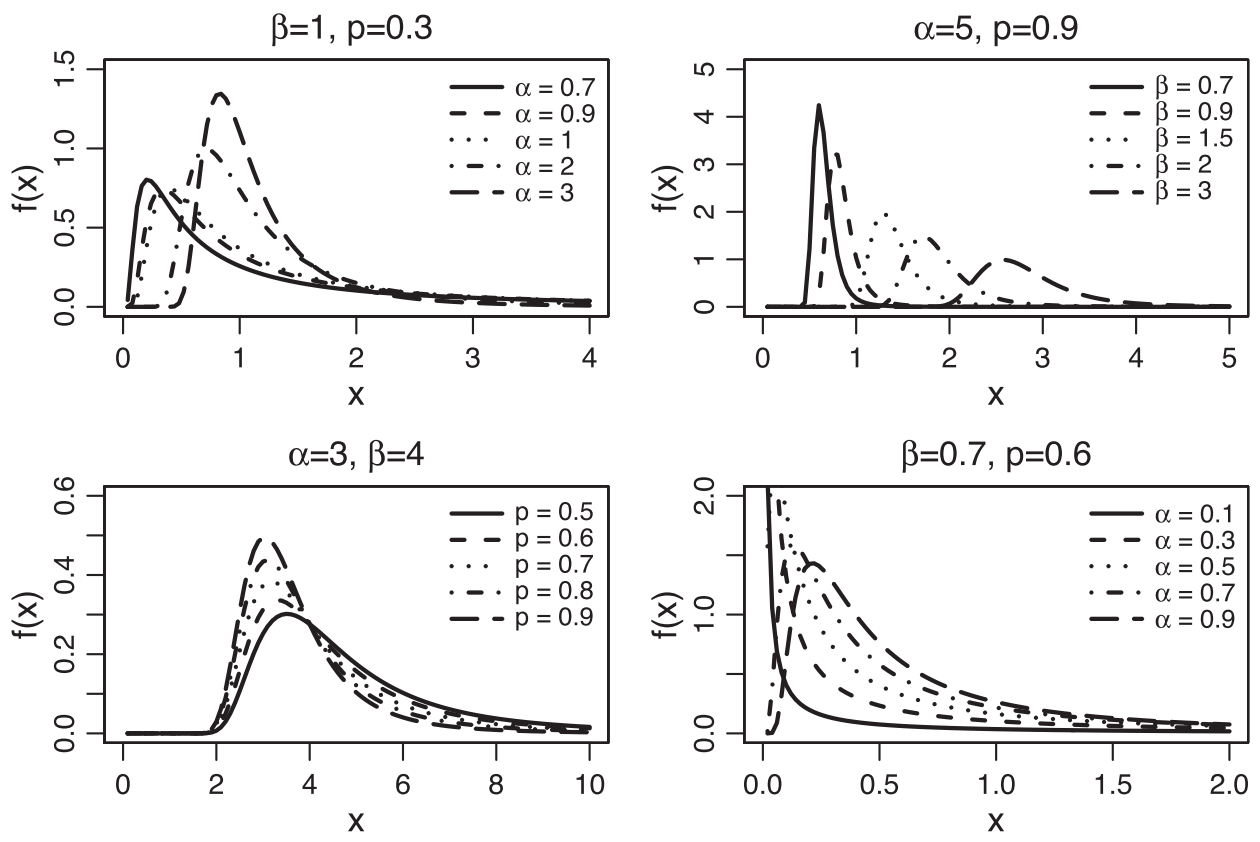

Figure 1 The probability density function plots for transmuted lower record type Fréchet (TLRT-F) distribution.
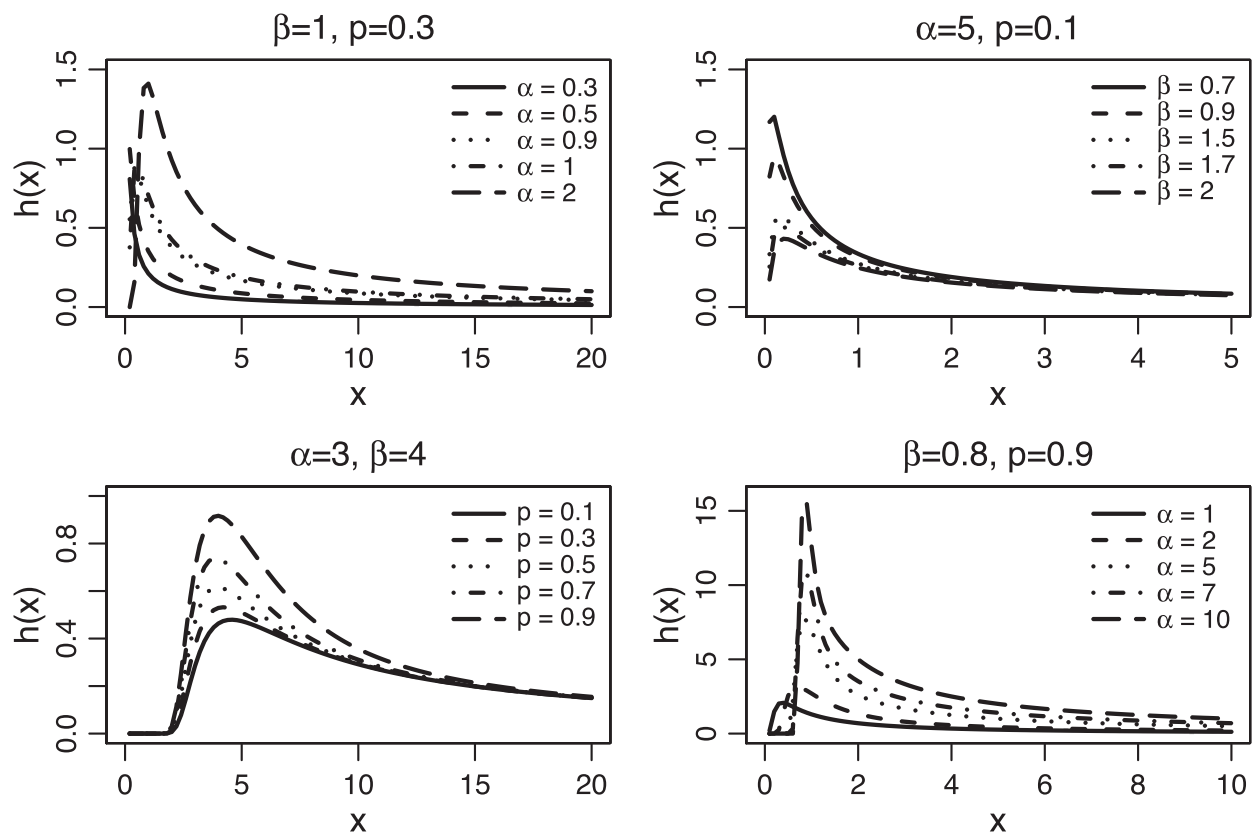

Figure 2 The hazard function plots of transmuted lower record type Fréchet (TLRT-F) distribution.

For $r \in \mathbb{N}_{+}$, the $r^{\text {th }}$ moment of TLRT-F distribution is given by

$$
E\left(X^{r}\right)=(1-p) \beta^{r} \Gamma\left(1-\frac{r}{\alpha}\right)+p \beta^{r} \Gamma\left(2-\frac{r}{\alpha}\right)
$$

where $\Gamma(\cdot)$ is a gamma function. Using (3.7), the mean and variance of TLRT-F distribution are obtained by

$$
E(X)=(1-p) \beta \Gamma\left(1-\frac{1}{\alpha}\right)+p \beta \Gamma\left(2-\frac{1}{\alpha}\right)
$$


and

$$
\begin{aligned}
\operatorname{Var}(X)= & (1-p) \beta^{2} \Gamma\left(1-\frac{2}{\alpha}\right)+p \beta^{2} \Gamma\left(2-\frac{2}{\alpha}\right) \\
& -\left\{(1-p) \beta \Gamma\left(1-\frac{1}{\alpha}\right)+p \beta \Gamma\left(2-\frac{1}{\alpha}\right)\right\}^{2},
\end{aligned}
$$

respectively. The moment generating function of TLRT-F distribution is also given by

$$
M(t)=(1-p) \sum_{r=0}^{\infty} \frac{t^{r}}{r !} \beta^{r} \Gamma\left(1-\frac{r}{\alpha}\right)+p \sum_{r=0}^{\infty} \frac{t^{r}}{r !} \beta^{r} \Gamma\left(2-\frac{r}{\alpha}\right) .
$$

The incomplete moments of TLRT-F distribution is obtained by

$$
m_{r}(y)=(1-p) \beta^{r} \Gamma\left(1-\frac{r}{\alpha},\left(\frac{\beta}{y}\right)^{\alpha}\right)+p \beta^{r} \Gamma\left(2-\frac{r}{\alpha},\left(\frac{\beta}{y}\right)^{\alpha}\right),
$$

where $\Gamma(a, x)$ is incomplete gamma function defined by

$$
\Gamma(a, x)=\int_{x}^{\infty} t^{a-1} e^{-t} d t
$$

The Bonferroni and Lorenz curves for TLRT-F are given, respectively, by

$$
B(\pi)=\frac{(1-p) \beta^{r} \Gamma\left(1-\frac{r}{\alpha},\left(\frac{\beta}{\nu}\right)^{\alpha}\right)+p \beta^{r} \Gamma\left(2-\frac{r}{\alpha},\left(\frac{\beta}{\nu}\right)^{\alpha}\right)}{\pi\left\{(1-p) \beta \Gamma\left(1-\frac{1}{\alpha}\right)+p \beta \Gamma\left(2-\frac{1}{\alpha}\right)\right\}},
$$

and

$$
L(\pi)=\frac{(1-p) \beta^{r} \Gamma\left(1-\frac{r}{\alpha},\left(\frac{\beta}{\nu}\right)^{\alpha}\right)+p \beta^{r} \Gamma\left(2-\frac{r}{\alpha},\left(\frac{\beta}{\nu}\right)^{\alpha}\right)}{(1-p) \beta \Gamma\left(1-\frac{1}{\alpha}\right)+p \beta \Gamma\left(2-\frac{1}{\alpha}\right)},
$$

where $\nu=Q(\pi ; \theta)$ and $Q$ is quantile function defined in (3.6).

Stochastic and the other ordering are important means for evaluating the comparative properties for a positive continuous random variable. The following theorem shows that the TLRT-F random variables can be ordered with respect to the likelihood ratio.

Theorem 3.1. Let $X \sim T L R T-F\left(\alpha, \beta, p_{1}\right)$ and $Y \sim T L R T-F\left(\alpha, \beta, p_{2}\right)$. If $p_{1}<p_{2}$ then $X$ is smaller than $Y$ in the likelihood ratio order, i.e., the ratio function of the corresponding $p d f$ s is decreasing in $x$.

Proof. For any $x>0$, the ratio of the densities is given by

$$
g(x)=\frac{1-p_{1}+p_{1}\left(\frac{\beta}{x}\right)^{\alpha}}{1-p_{2}+p_{2}\left(\frac{\beta}{x}\right)^{\alpha}} .
$$

Consider the derivative of $\log (g(x))$ in $x$

$$
\frac{d \log (g(x))}{d x}=\frac{\alpha \beta^{\alpha}\left(p_{1}-p_{2}\right)}{x^{\alpha-1}\left(1-p_{1}+p_{1}\left(\frac{\beta}{x}\right)^{\alpha}\right)\left(1-p_{2}+p_{2}\left(\frac{\beta}{x}\right)^{\alpha}\right)}<0
$$

for $p_{1}<p_{2}$ and hence proof is completed.

Corollary 3.1. It follows from [8] that $X$ is also smaller than $Y$ in the hazard ratio, mean residual life and stochastic orders under the conditions given in Theorem 3.1.

In order to generate the data from TLRT-F distribution, an acceptance-rejection (AR) sampling method is given in the following algorithm. In this algorithm, the Weibull distribution is chosen as a proposal distribution. The AR algorithm is given as follows: 


\section{Algorithm 1 :}

1 Generate data on random variable $Y$ from Weibull distribution with pdf $g$ given as follows:

$$
g(z ; \alpha, \beta)=\frac{\alpha}{\beta}\left(\frac{y}{\beta}\right)_{z}^{\alpha-1} \exp \left(-\left(\frac{y}{\beta}\right)_{z}^{\alpha}\right) .
$$

2 Generate $U$ from standard uniform distribution(independent of $Y$ ).

3 If

$$
U<\frac{f(Y ; \boldsymbol{\theta})}{k \times g(Y ; \alpha, \beta)}
$$

then set $X=Y$ ("accept"); otherwise go back to Al ("reject"), where pdf $f$ is given as in (3.4) and

$$
k=\max _{z \in \mathbb{R}_{+}} \frac{f(z ; \boldsymbol{\theta})}{g(z ; \alpha, \beta)} .
$$

The output of this algorithm suggests a random data on $X$ from TLRT-F $(\boldsymbol{\theta})$. It is noticed that the Algorithm 1 is used for all simulations in the paper.

\section{STATISTICAL INFERENCE ON DISTRIBUTION PARAMETERS}

In this section, we propose five estimators to estimate the unknown parameters of the TLRT-F $(\boldsymbol{\theta})$ distribution. They are the maximum likelihood, least squares, weighted least squares, Cramér-von Mises type and Anderson-Darling type estimates. A simulation study is performed to observe the performances of the methods discussed here.

\subsection{Point Estimation}

Let $x_{1}, x_{2}, \ldots, x_{n}$ be a realization of a random sample from the TLRT-F $(\boldsymbol{\theta})$ distribution and $x_{(1)}<x_{(2)}<\cdots<x_{(n)}$ denotes the corresponding observed order statistics. Then, the log-likelihood function is written by

$$
\begin{aligned}
\ell(\theta)= & n \log (\alpha)+n \alpha \log (\beta)-(\alpha+1) \sum_{i=1}^{n} \log \left(x_{i}\right) \\
& -\sum_{i=1}^{n}\left(\frac{\beta}{x_{i}}\right)^{\alpha}+\sum_{i=1}^{n} \log \left(1-p+p\left(\frac{\beta}{x_{i}}\right)^{\alpha}\right) .
\end{aligned}
$$

Hence, the maximum likelihood estimate (MLE) of $\boldsymbol{\theta}$ is given by

$$
\hat{\theta}_{1}=\underset{\theta}{\arg \max }\{\ell(\theta)\}
$$

where $\boldsymbol{\theta}=(\alpha, \beta, p)$ is the parameter vector and $\hat{\boldsymbol{\theta}}_{1}=(\hat{\alpha}, \hat{\beta}, \hat{p})$ is MLE of $\boldsymbol{\theta}$. Let us define the following functions which are used to define the different types of estimators:

$$
\begin{aligned}
Q_{L S}(\boldsymbol{\theta}) & =\sum_{i=1}^{n}\left(F\left(x_{(i)}\right)-\frac{i}{n+1}\right)^{2}, \\
Q_{W L S}(\boldsymbol{\theta}) & =\sum_{i=1}^{n} \frac{(n+2)(n+1)^{2}}{i(n-i+1)}\left(F\left(x_{(i)}\right)-\frac{i}{n+1}\right)^{2}, \\
Q_{C v M}(\boldsymbol{\theta}) & =\frac{1}{12 n}+\sum_{i=1}^{n}\left(F\left(x_{(i)}\right)-\frac{2 i-1}{2 n}\right)^{2},
\end{aligned}
$$

and

$$
Q_{A D}(\boldsymbol{\theta})=-n-\frac{1}{n} \sum_{i=1}^{n}\left\{(2 i-1) \log \left(F\left(x_{(i)}\right)\right)\right\}+\frac{1}{n} \sum_{i=1}^{n} \log \left\{1-F\left(x_{(i)}\right)\right\},
$$

where $F(\cdot)$ is cdf of TLRT-F $(\boldsymbol{\theta})$ distribution given in (3.3). Then, the least squares estimator (LSE), weighted least squares estimator (WLSE), Anderson-Darling estimator (ADE) and the Cramér-von Mises estimator (CvME) of $\boldsymbol{\theta}$ are given, respectively, by

$$
\hat{\boldsymbol{\theta}}_{2}=\underset{\boldsymbol{\theta}}{\arg \min }\left\{Q_{L S}(\boldsymbol{\theta})\right\},
$$




$$
\begin{gathered}
\hat{\boldsymbol{\theta}}_{3}=\underset{\boldsymbol{\theta}}{\arg \min }\left\{Q_{W L S}(\boldsymbol{\theta})\right\}, \\
\hat{\boldsymbol{\theta}}_{4}=\underset{\boldsymbol{\theta}}{\arg \min }\left\{Q_{A D}(\boldsymbol{\theta})\right\}, \\
\hat{\boldsymbol{\theta}}_{5}=\underset{\boldsymbol{\theta}}{\arg \min }\left\{Q_{C v M}(\boldsymbol{\theta})\right\} .
\end{gathered}
$$

The optimization problems can be solved by some numerical methods such as Nelder-Mead, BFGS, L-BFGS-B or CG. These methods can be easily employed by optim function in R.

\subsection{Simulation Study for Point Estimates}

In the simulation study, 5000 trials are used to estimates the bias and MSE of the MLEs, LSEs, WLSEs, ADEs and CVMEs. The sample sizes are considered as $n=50,100,250,500,750$ and 1000. Two parameter settings are considered. The results are given in the Tables 1 and 2. In the simulation, the samples are generated from TLRT-F $(\boldsymbol{\theta})$ distribution by using AR sampling given in Algorithm 1. The numerical methods BFGS, Nelder-Mead, CG and L-BFGS-B are used to achieve the values of estimates by optimizing the objective functions (4.1)-(4.5). The values obtained from the numerical method that optimizes the objective functions are selected as parameter estimates. The bias and MSEs of estimators are presented in Tables 1 and 2. From Tables 1 and 2, it is observed that the bias and MSEs of the all estimators decrease to zero as expected. The WLSEs and ADEs have similar MSEs for all sample of size and they are better than the others. The MSE of MLEs tends to MSE of WLSEs and ADEs for large sample size.

\section{TLRT-F REGRESSION ANALYSIS}

The log-location-scale regression models are studied by several authors such as [9-12]. In this section, we describe the usage of log-location-

\begin{tabular}{|c|c|c|c|c|c|c|c|}
\hline \multirow[b]{2}{*}{$n$} & & \multicolumn{3}{|c|}{ Bias } & \multicolumn{3}{|c|}{ MSE } \\
\hline & & $\alpha$ & $\beta$ & $p$ & $\alpha$ & $\beta$ & $p$ \\
\hline \multirow[t]{6}{*}{ MLEs } & 50 & 0.0986 & -0.0534 & -0.1802 & 0.0367 & 0.0183 & 0.0995 \\
\hline & 100 & 0.0587 & -0.0299 & -0.1155 & 0.0234 & 0.0155 & 0.0803 \\
\hline & 250 & 0.0333 & -0.0155 & -0.0761 & 0.0158 & 0.0126 & 0.0648 \\
\hline & 500 & 0.0235 & -0.0113 & -0.0585 & 0.0115 & 0.0097 & 0.0503 \\
\hline & 750 & 0.0243 & -0.0139 & -0.0573 & 0.0095 & 0.0078 & 0.0421 \\
\hline & 1000 & 0.0209 & -0.0123 & -0.0517 & 0.0084 & 0.0069 & 0.0385 \\
\hline \multirow[t]{6}{*}{ LSEs } & 50 & -0.0028 & 0.0326 & -0.0241 & 0.0318 & 0.0442 & 0.0645 \\
\hline & 100 & 0.0049 & 0.0139 & -0.0259 & 0.0214 & 0.0223 & 0.0614 \\
\hline & 250 & 0.0044 & 0.0120 & -0.0165 & 0.0155 & 0.0152 & 0.0455 \\
\hline & 500 & 0.0129 & -0.0014 & -0.0334 & 0.0112 & 0.0103 & 0.0402 \\
\hline & 750 & 0.0140 & -0.0034 & -0.0311 & 0.0094 & 0.0080 & 0.0314 \\
\hline & 1000 & 0.0126 & -0.0030 & -0.0293 & 0.0087 & 0.0071 & 0.0294 \\
\hline \multirow[t]{6}{*}{ WLSEs } & 50 & 0.0150 & 0.0031 & -0.0598 & 0.0251 & 0.0231 & 0.0590 \\
\hline & 100 & 0.0142 & 0.0024 & -0.0410 & 0.0179 & 0.0161 & 0.0503 \\
\hline & 250 & 0.0102 & 0.0029 & -0.0284 & 0.0125 & 0.0113 & 0.0404 \\
\hline & 500 & 0.0122 & -0.0022 & -0.0314 & 0.0094 & 0.0083 & 0.0333 \\
\hline & 750 & 0.0146 & -0.0060 & -0.0336 & 0.0077 & 0.0065 & 0.0280 \\
\hline & 1000 & 0.0135 & -0.0057 & -0.0322 & 0.0072 & 0.0059 & 0.0264 \\
\hline \multirow[t]{6}{*}{ ADEs } & 50 & 0.0205 & 0.0078 & -0.0548 & 0.0254 & 0.0267 & 0.0562 \\
\hline & 100 & 0.0183 & -0.0027 & -0.0467 & 0.0162 & 0.0139 & 0.0533 \\
\hline & 250 & 0.0114 & 0.0021 & -0.0308 & 0.0127 & 0.0113 & 0.0436 \\
\hline & 500 & 0.0104 & -0.0007 & -0.0277 & 0.0092 & 0.0082 & 0.0332 \\
\hline & 750 & 0.0121 & -0.0036 & -0.0282 & 0.0075 & 0.0064 & 0.0268 \\
\hline & 1000 & 0.0118 & -0.0040 & -0.0284 & 0.0070 & 0.0058 & $\begin{array}{r}0.0258 \\
\text { ttinued) }\end{array}$ \\
\hline
\end{tabular}
scale TLRT-F regression analysis.

Table 1 Average bias and MSEs of the estimates for the true parameters $\boldsymbol{\theta}=(1,0.5,0.7)$. 
Table 1 Average bias and MSEs of the estimates for the true parameters $\theta=(1,0.5,0.7)$. (Continued)

\begin{tabular}{|c|c|c|c|c|c|c|c|}
\hline \multirow[b]{2}{*}{$n$} & & \multicolumn{3}{|c|}{ Bias } & \multicolumn{3}{|c|}{ MSE } \\
\hline & & $\alpha$ & $\beta$ & $p$ & $\alpha$ & $\beta$ & $p$ \\
\hline \multirow[t]{6}{*}{ CvMEs } & 50 & 0.0261 & 0.0320 & -0.0211 & 0.0371 & 0.0469 & 0.0738 \\
\hline & 100 & 0.0195 & 0.0134 & -0.0254 & 0.0240 & 0.0237 & 0.0669 \\
\hline & 250 & 0.0105 & 0.0115 & -0.0172 & 0.0165 & 0.0157 & 0.0482 \\
\hline & 500 & 0.0163 & -0.0018 & -0.0343 & 0.0118 & 0.0105 & 0.0417 \\
\hline & 750 & 0.0164 & -0.0038 & -0.0321 & 0.0098 & 0.0082 & 0.0325 \\
\hline & 1000 & 0.0145 & -0.0034 & -0.0302 & 0.0090 & 0.0073 & 0.0302 \\
\hline
\end{tabular}

MSE, mean squared error; MLE, maximum likelihood estimate; LSE, least squares estimator; WLSE, weighted least squares estimator; ADE, Anderson Darling estimator; CvME, Cramér-von Mises estimator.

Table 2 Average bias and MSEs of the estimates for the true parameters $\boldsymbol{\theta}=(3,2,0.9)$.

\begin{tabular}{|c|c|c|c|c|c|c|c|}
\hline \multirow[b]{2}{*}{$n$} & & \multicolumn{3}{|c|}{ Bias } & \multicolumn{3}{|c|}{ MSE } \\
\hline & & $\alpha$ & $\beta$ & $p$ & $\alpha$ & $\beta$ & $p$ \\
\hline \multirow[t]{6}{*}{ MLEs } & 50 & 0.5643 & -0.1473 & -0.2707 & 0.6119 & 0.0448 & 0.1357 \\
\hline & 100 & 0.3529 & -0.0996 & -0.1791 & 0.3561 & 0.0305 & 0.0899 \\
\hline & 250 & 0.1590 & -0.0445 & -0.0820 & 0.1522 & 0.0151 & 0.0440 \\
\hline & 500 & 0.0748 & -0.0203 & -0.0400 & 0.0759 & 0.0081 & 0.0230 \\
\hline & 750 & 0.0483 & -0.0126 & -0.0242 & 0.0485 & 0.0053 & 0.0144 \\
\hline & 1000 & 0.0317 & -0.0076 & -0.0139 & 0.0325 & 0.0036 & 0.0087 \\
\hline \multirow[t]{6}{*}{ LSEs } & 50 & 0.3154 & -0.0816 & -0.1645 & 0.4937 & 0.0476 & 0.1010 \\
\hline & 100 & 0.2540 & -0.0614 & -0.1239 & 0.3698 & 0.0361 & 0.0813 \\
\hline & 250 & 0.1667 & -0.0378 & -0.0787 & 0.2271 & 0.0219 & 0.0553 \\
\hline & 500 & 0.0999 & -0.0229 & -0.0496 & 0.1338 & 0.0132 & 0.0340 \\
\hline & 750 & 0.0720 & -0.0164 & -0.0347 & 0.0940 & 0.0093 & 0.0242 \\
\hline & 1000 & 0.0474 & -0.0100 & -0.0217 & 0.0672 & 0.0068 & 0.0169 \\
\hline \multirow[t]{6}{*}{ WLSEs } & 50 & 0.3550 & -0.1027 & -0.1956 & 0.4285 & 0.0402 & 0.0995 \\
\hline & 100 & 0.2705 & -0.0747 & -0.1384 & 0.3017 & 0.0284 & 0.0684 \\
\hline & 250 & 0.1533 & -0.0398 & -0.0753 & 0.1610 & 0.0157 & 0.0404 \\
\hline & 500 & 0.0813 & -0.0208 & -0.0414 & 0.0869 & 0.0088 & 0.0226 \\
\hline & 750 & 0.0528 & -0.0130 & -0.0251 & 0.0546 & 0.0055 & 0.0136 \\
\hline & 1000 & 0.0327 & -0.0074 & -0.0141 & 0.0360 & 0.0038 & 0.0083 \\
\hline \multirow[t]{6}{*}{ ADEs } & 50 & 0.3749 & -0.1044 & -0.1930 & 0.4107 & 0.0361 & 0.1043 \\
\hline & 100 & 0.2808 & -0.0775 & -0.1377 & 0.2808 & 0.0254 & 0.0620 \\
\hline & 250 & 0.1515 & -0.0391 & -0.0721 & 0.1495 & 0.0143 & 0.0367 \\
\hline & 500 & 0.0796 & -0.0202 & -0.0394 & 0.0809 & 0.0082 & 0.0208 \\
\hline & 750 & 0.0525 & -0.0129 & -0.0245 & 0.0528 & 0.0054 & 0.0133 \\
\hline & 1000 & 0.0330 & -0.0074 & -0.0138 & 0.0352 & 0.0037 & 0.0082 \\
\hline \multirow[t]{6}{*}{ CvMEs } & 50 & 0.4034 & -0.0813 & -0.1560 & 0.6166 & 0.0502 & 0.1073 \\
\hline & 100 & 0.2947 & -0.0611 & -0.1193 & 0.4201 & 0.0375 & 0.0853 \\
\hline & 250 & 0.1814 & -0.0376 & -0.0767 & 0.2425 & 0.0225 & 0.0574 \\
\hline & 500 & 0.1064 & -0.0227 & -0.0483 & 0.1395 & 0.0135 & 0.0350 \\
\hline & 750 & 0.0757 & -0.0161 & -0.0335 & 0.0965 & 0.0094 & 0.0247 \\
\hline & 1000 & 0.0498 & -0.0097 & -0.0206 & 0.0684 & 0.0069 & 0.0171 \\
\hline
\end{tabular}

MSE, mean squared error; MLE, maximum likelihood estimate; LSE, least squares estimator; WLSE, weighted least squares estimator; ADE, Anderson Darling estimator; CvME, Cramér-von Mises estimator.

Let $X$ be a TLRT-F $(\theta)$ random variable. Let us consider a reparameterization by $\beta=\exp (\mu)$ and $\alpha=1 / \sigma$. Thus, the $\log$-lifetime $Y=\log (X)$ is a random variable with the pdf and cdf

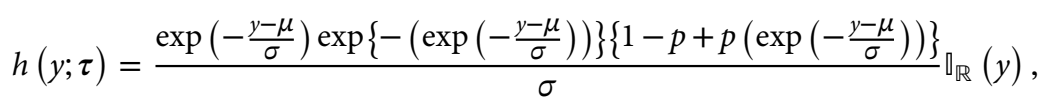


and

$$
H(y ; \tau)=\exp \left\{-\left(\exp \left(-\frac{y-\mu}{\sigma}\right)\right)\right\}\left\{1+p\left(\exp \left(-\frac{y-\mu}{\sigma}\right)\right)\right\}
$$

where $\tau=(\mu, \sigma, p), \mu$ and $\sigma$ are location and scale parameters, respectively.

The distribution of $Y$ with $c d f(5.2)$ is denoted $\log$-TLRT-F $(\mu, \sigma, p)$. Let us consider the regression model

$$
\mathbf{Y}=\mu+\sigma \varepsilon
$$

where $Y_{1}, Y_{2}, \ldots, Y_{n}$ are random sample from $\log$-TLRT-F $\left(\mu_{i}=Z_{i}^{\mathrm{T}} \boldsymbol{\beta}, \sigma, p\right)$ distribution, and $\mathbf{Y}=\left(Y_{1}, Y_{2}, \ldots, Y_{n}\right)^{\mathrm{T}}$. Furthermore, $\boldsymbol{\beta}=\left(\beta_{1}, \ldots, \beta_{p}\right)^{\mathrm{T}}, \boldsymbol{\mu}=\left(\mu_{1}, \ldots, \mu_{n}\right)^{\mathrm{T}}, \boldsymbol{\varepsilon}=\left(\varepsilon_{1}, \ldots, \varepsilon_{n}\right)^{\mathrm{T}}, \mu_{i}=\boldsymbol{Z}_{i}^{\mathrm{T}} \boldsymbol{\beta}$ and $\boldsymbol{Z}_{i}=\left(Z_{i 1}, \ldots, Z_{i q}\right)^{\mathrm{T}}$ are $i$ th values of covariates for $i=1,2, \ldots, n$. In addition, $\varepsilon_{i}=\left(Y_{i}-\mu_{i}\right) / \sigma$ for $i=1,2, \ldots, n$ is a random error from log-TLRT-F $(\mu=0, \sigma=1, p)$. In this model, the location parameter is assumed as a linear function of covariates $\boldsymbol{Z}_{i}$.

Let us discuss the MLEs of parameters $\eta=(\beta, \sigma, p)$ in the model (5.3) under Type-I right censoring. Suppose that the log lifetimes $Y_{i}(i=1,2, \ldots, n)$ are Type-I right censored $\left(\right.$ at $\left.\log \left(c_{i}\right)\right)$ from $\log$-TLRT-F $\left(\mu_{i}, \sigma, p\right)$, where $c_{i}$ is censoring time for lifetime $X_{i}$. Let us define

$$
T_{i}=\min \left\{Y_{i}, \log \left(c_{i}\right)\right\}, i=1,2, \ldots, n .
$$

Hence, the log-likelihood function based on the Type-I right censored sample $T_{1}, T_{2}, \ldots, T_{n}$ is written by

$$
\ell(\eta)=\sum_{i=1}^{n}\left\{\omega_{i} \log \left(h\left(t_{i} ;\left(\boldsymbol{Z}_{i}^{\mathrm{T}} \boldsymbol{\beta}, \sigma, p\right)\right)\right)+\left(1-\omega_{i}\right) \log \left(1-H\left(t_{i} ;\left(\boldsymbol{Z}_{i}^{\mathrm{T}} \boldsymbol{\beta}, \sigma, p\right)\right)\right)\right\},
$$

where

$$
\omega_{i}=\left\{\begin{array}{l}
0, T_{i}>\log \left(c_{i}\right) \\
1, T_{i} \leq \log \left(c_{i}\right)
\end{array}\right.
$$

is an indicator function and $t_{i}$ denotes the observed value of $T_{i}, i=1,2, \ldots, n$.

The MLE of $\eta$ can be obtained by maximizing the log-likelihood given in (5.4). Some numerical methods such as Nelder-Mead, BFGS, CG or L-BFGS-B can be used for the maximization problem. These methods are available in $\mathrm{R}$.

\section{REAL DATA APPLICATION}

In this section, we report a data modeling analysis for the glass fibers data. For the comparison issue, we consider TLRT-F, Fréchet (Fr), transmuted Log-logistic (TLL) [13], Weibull (W), exponentiated Exponential (EE) [14], transmuted Weibull (TW) [15], Lindley (L), Gompertz (G) distributions. The pdf of these distributions are given in Table 3. Table 4 presents the MLEs (standard errors) and Table 5 contains $-2 \times \log$-likelihood value, Akaike's information criteria (AIC), Kolmogorov-Smirnov test statistic (KS), Anderson-Darling statistic ( $A^{*}$ ), Cramer von-Mises statistic (CVM) and $p$ values based on these statistics for the all distributions given in Table 3. Figure 3 shows that the fitted cdfs for glass fibers data. According to results in Table 5, it is observed that the TLRT-F has minimum KS, $\mathrm{A}^{*}$ and CVM values. Hence, it can be said that the TLRT-F can be a good alternative to modeling real data.

\section{Glass fibers data}

This data set is generated data to simulate the strengths of glass fibers in Mahmoud and Mandouh [16]. The data are as follows: 1.014, 1.081, $1.082,1.185,1.223,1.248,1.267,1.271,1.272,1.275,1.276,1.278,1.286,1.288,1.292,1.304,1.306,1.355,1.361,1.364,1.379,1.409,1.426$, $1.459,1.46,1.476,1.481,1.484,1.501,1.506,1.524,1.526,1.535,1.541,1.568,1.579,1.581,1.591,1.593,1.602,1.666,1.67,1.684,1.691$, $1.704,1.731,1.735,1.747,1.748,1.757,1.800,1.806,1.867,1.876,1.878,1.91,1.916,1.972,2.012,2.456,2.592,3.197,4.121$.

\section{LIFETIME REGRESSION ANALYSIS WITH REAL DATA}

Lawless [17] reported the failure times for epoxy insulation specimens in an accelerated voltage life test. The sample size is $n=60$ and there are three levels of voltage 52.5, 55.0 and 57.5. Let $y_{i}$ be failure times for epoxy insulation specimens (in min) and $v_{i 1}$ are voltages (kV). The following regression model can be written by

$$
y_{i}=\beta_{0}+\beta_{1} v_{i 1}+\sigma z_{i}
$$


Table 3 List of the lifetime distribution to modeling real data.

\begin{tabular}{ll}
\hline$f_{F r}(\alpha, \beta)=\alpha \beta^{\alpha} x^{-(\alpha+1)} \exp \left(-\left(\frac{\beta}{x}\right)^{\alpha}\right)$, & $\alpha, \beta>0$ \\
$f_{T L L}(\alpha, \beta, \lambda)=\frac{e^{\alpha} x^{\beta-1}\left(\left(1+e^{\alpha} x^{\beta}\right)-\lambda\left(e^{\alpha} x^{\beta}-1\right)\right)}{\left(1+e^{\alpha} x^{\beta}\right)^{3}}, \alpha$ & $\alpha, \beta>0, \lambda \in[-1,1]$ \\
$f_{W}(\alpha, \beta)=\frac{\alpha}{\beta}\left(\frac{x}{\beta}\right)^{\alpha-1} \exp \left(-\left(\frac{x}{\beta}\right)^{\alpha}\right)$, & $\alpha, \beta>0$ \\
$f_{E E}(\theta, \alpha)=\theta \alpha e^{-\alpha x}\left(1-e^{-\alpha x}\right)^{\theta-1}$, & $\alpha, \theta>0$ \\
$f_{T W}(\alpha, \beta, \lambda)=\frac{\alpha}{\beta}\left(\frac{x}{\beta}\right)^{\alpha-1} \exp \left(-\left(\frac{x}{\beta}\right)^{\alpha}\right)\left(1+\lambda-2 \lambda \exp \left(-\left(\frac{x}{\beta}\right)^{\alpha}\right)\right)$, & $\alpha, \beta>0, \lambda \in[-1,1]$ \\
$f_{L}(\theta)=\frac{\theta^{2}}{\theta+1}(1+x) e^{-\theta x}$, & $\theta>0$ \\
$f_{G}(a, b)=a e^{b x} \exp \left(-\frac{a}{b}\left(e^{b x}-1\right)\right)$, & $a, b>0$ \\
\hline
\end{tabular}

Table 4 MLEs (standard errors) for glass fibers data.

\begin{tabular}{ll}
\hline Distribution & MLEs \\
\hline TLRT-F & $\hat{\alpha}=4.0908(0.5563), \hat{\beta}=1.6764(0.0879), \hat{p}=0.8609(0.1689)$ \\
Fr & $\hat{\alpha}=5.4378(0.5192), \hat{\beta}=1.4108(0.0344)$ \\
TLL & $\hat{\alpha}=1.6103(0.1265), \hat{\beta}=8.0742(0.0704), \hat{\lambda}=0.4056(0.6049)$ \\
W & $\hat{\alpha}=3.0620(0.2403), \hat{\beta}=1.7875(0.0784)$ \\
EE & $\hat{\theta}=165.9968(84.3295), \hat{\alpha}=3.5557(0.3665)$ \\
TW & $\hat{\alpha}=3.4505(0.2645), \hat{\beta}=2.0788(0.0868), \hat{\lambda}=0.8512(0.1146)$ \\
L & $\hat{\theta}=0.9459(0.0931)$ \\
G & $\hat{a}=0.1791(0.0407), \hat{b}=1.0508(0.1173)$ \\
\hline
\end{tabular}

MLE, maximum likelihood estimate; TLRT-F, transmuted lower record type Fréchet; TLL, transmuted Loglogistic; W, Weibull, EE, exponentiated exponential; TW, transmuted Weibull; L, Lindley; G, Gompertz.

Table 5 Selection criteria statistics for glass fibers data.

\begin{tabular}{|c|c|c|c|c|c|c|c|c|}
\hline TLRT-F & 38.9698 & 44.9698 & 0.0662 & 0.9283 & 0.4285 & 0.8195 & 0.0563 & 0.8389 \\
\hline Fr & 40.1277 & 44.1277 & 0.0772 & 0.8187 & 0.5291 & 0.7167 & 0.0699 & 0.7540 \\
\hline $\mathrm{W}$ & 92.7338 & 96.7338 & 0.2051 & 0.0084 & 5.2609 & 0.0022 & 0.8853 & 0.0044 \\
\hline $\mathrm{L}$ & 170.9594 & 172.9594 & 0.4387 & $1.50 \mathrm{E}-11$ & 15.8383 & $9.52 \mathrm{E}-06$ & 3.3144 & 5.11E-09 \\
\hline $\mathrm{EE}$ & 45.1408 & 49.1408 & 0.0717 & 0.8794 & 0.6638 & 0.5892 & 0.0724 & 0.7385 \\
\hline
\end{tabular}

MLE, maximum likelihood estimate; TLRT-F, transmuted lower record type Fréchet; TLL, transmuted Log-logistic; W, Weibull, EE, exponentiated exponential; TW, transmuted Weibull; L, Lindley; G, Gompertz; AIC, Akaike’s information criteria; KS, Kolmogorov-Smirnov test; A*, Anderson Darling statistic; CVM, Cramer von-Mises.

where the realization $y_{i}$ is assumed an observation from LTLRT-F distribution given in (5.1). For comparison, the LW (see, Lawless [17]) and LTLGBXII (see, Yousof et al. [9]) lifetime regression models are considered. The results of analyses are presented in Table 6. To compare LTLRT-F with LW and LTLGBXII regression models, the MLEs of the model parameters, the asymptotic standard errors of these estimates and the values of the minus log-likelihood and AIC measures are given in Table 6. It is noticed that the results for LW and LTLGBXII are reproduced from Yousof et al. [9]. Figure 4 exhibit, the fitted and empirical survival function plots. From Figure 4 and Table 6, one can conclude that the fitted LTLRT-F regression model can be strongly recommended for modeling survival data given in the literature.

\section{CONCLUSION}

In this study, a new extension to generate a new family of distribution is introduced by a transmuted lower record type map of order 2 . This method will be lead to obtain new families of distribution called TLRTs. A sub-model of this family is considered and lifetime regression analysis is introduced based on this sub-model. The introduced regression model is applied to a real data set and the results show that our model is a good alternative to modeling real data. 


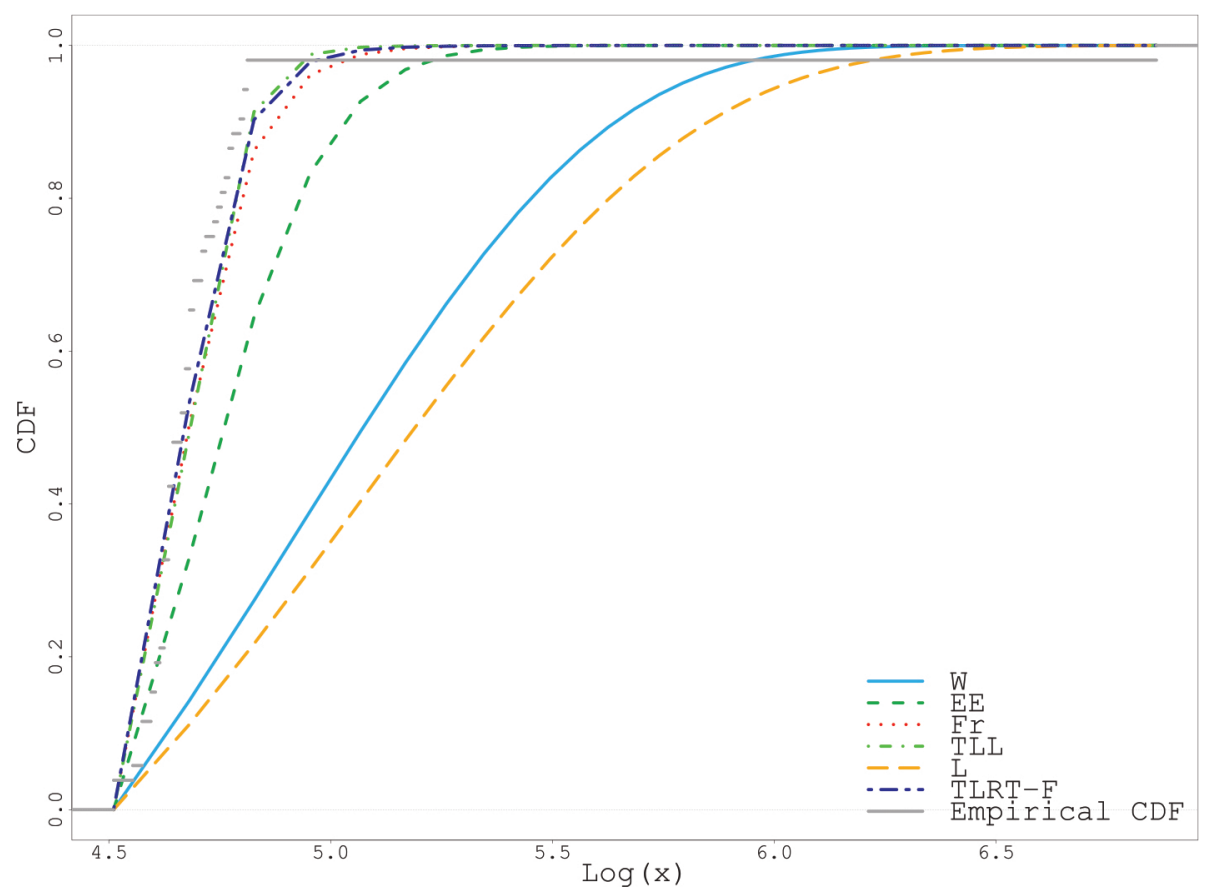

Figure 3 Fitted CDFs for glass fibers data.

Table 6 MLEs of the parameters, standard errors in second line, $p$-values in $[\cdot]$ and the AIC statistics.

\begin{tabular}{|c|c|c|c|c|c|c|c|c|}
\hline Model & $\beta_{0}$ & $\beta_{1}$ & $\sigma$ & $p$ & & & $-\ell$ & $A I C$ \\
\hline \multirow[t]{2}{*}{ LTLRT-F } & $\begin{array}{c}15.6527 \\
3.5915 \\
{[0.0000]}\end{array}$ & $\begin{array}{c}-0.1564 \\
0.0625 \\
{[0.0123]}\end{array}$ & $\begin{array}{l}1.2378 \\
0.2637\end{array}$ & $\begin{array}{l}0.9880 \\
0.3261\end{array}$ & & & 78.278 & 164.556 \\
\hline & $\beta_{0}$ & $\beta_{1}$ & $\sigma$ & $\gamma$ & $\theta$ & $\beta$ & $-\ell$ & $A I C$ \\
\hline \multirow[t]{2}{*}{ LTLGBXII } & $\begin{array}{c}14.4513 \\
4.876\end{array}$ & $\begin{array}{c}-0.1790 \\
0.074\end{array}$ & $\begin{array}{c}0.8024 \\
0.827\end{array}$ & $\begin{array}{c}0.6860 \\
0.685\end{array}$ & $\begin{array}{c}7.7247 \\
7.027\end{array}$ & $\begin{array}{c}0.7089 \\
0.984\end{array}$ & 78.2 & 168.4 \\
\hline & $\beta_{0}$ & $\beta_{1}$ & $\sigma$ & & & & $-e$ & $A I C$ \\
\hline LW & $\begin{array}{c}22.0313 \\
3.0454\end{array}$ & $\begin{array}{c}-0.2745 \\
0.0553\end{array}$ & $\begin{array}{l}0.8453 \\
0.0904\end{array}$ & & & & 83.699 & 173.398 \\
\hline
\end{tabular}

MLE, maximum likelihood estimate; AIC, Akaike's information criteria.

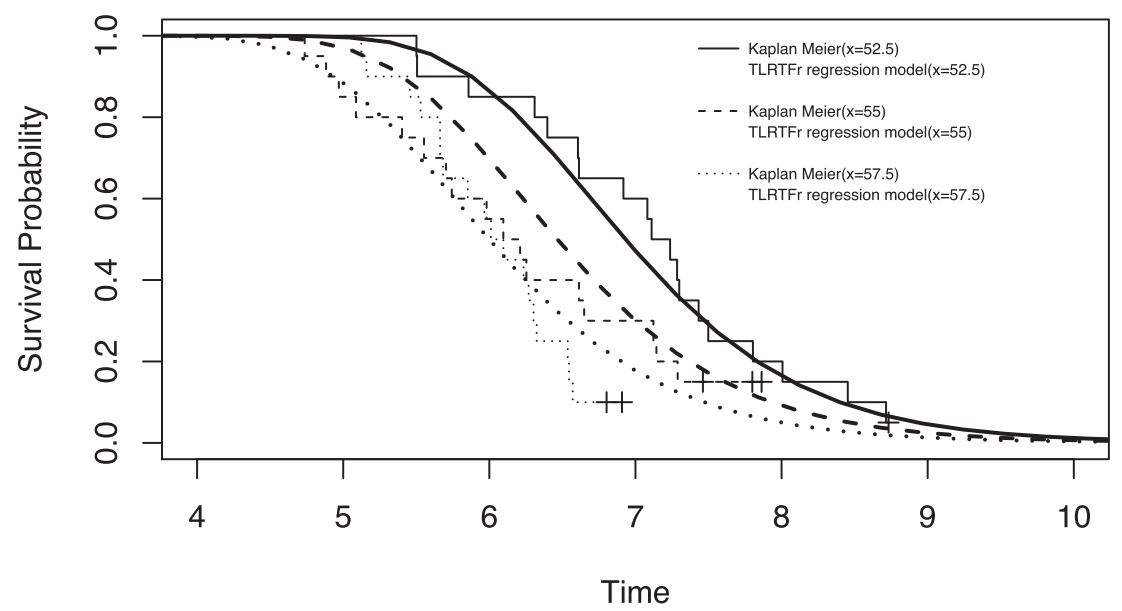

Figure 4 Fitted and empirical survival functions plots. 


\section{CONFLICTS OF INTEREST}

There is no conflict of interest.

\section{AUTHORS' CONTRIBUTIONS}

The authors thank the relevant editor and reviewer for their valuable suggestions, which were very useful in improving the study.

\section{Funding Statement}

This study is not supported by any funding council.

\section{ACKNOWLEDGMENTS}

We thank to Dr. Emrah Altun for his constructive comments on regression part of the study.

\section{REFERENCES}

1. W.T. Shaw, I.R. Buckley, The Alchemy of Probability Distributions: Beyond Gram Charlier \& Cornish Fisher Expansions, and Skew-Normalor Kurtotic-Normal Distributions, Technical report, Financial MathematicsGroup, King's College, London, U.K., 2007 , p. 64.

2. W.T. Shaw, I.R. Buckley, The alchemy of probability distributions: Beyond Gram-Charlier expansions, and a skew-kurtotic-normal distribution from a rank transmutation, map. 2009. http://arxiv.org/abs/0901.0434

3. M. Alizadeh, F. Merovci, G.G. Hamedani, Generalized transmuted family of distributions: properties and applications. Hacet. J. Math. Stat. 46 (2017), 645-667.

4. M. Aslam, Z. Hussain, Z. Asghar, Stoch. Qual. Control. 33 (2018), 103-112.

5. D.C.T. Granzotto, F. Louzada, N. Balakrishnan, J. Stat. Comput. Simul. 87 (2017), 2760-2778.

6. N. Balakrishnan, M. He, A Record-Based Transmuted Model, 2019. Under review, Advance Online Publication.

7. C. Tanış, B. Saraçoğlu, On the record-based transmuted model of Balakrishnan and He based on weibull distribution, Commun. Stat. Simul. Comput. (2020).

8. M. Shaked, J.G. Shanthikumar, Stochastic Orders and their Applications, Academic Press, London, England, 1994.

9. H.M. Yousof, E. Altun, M. Rasekhi, M. Alizadeh, G.G. Hamedan, M.M. Ali, Commun. Stat. Simul. Comput. 48 (2019), $264-286$.

10. E. Altun, H.M. Yousof, G.G. Hamedani, A new log-location regression model with influence diagnostics and residual analysis. Facta Universitatis, Series: Mathematics and Informatics, 33(3), (2018), 417-449.

11. A. Pekgör, C. Kus, K. Karakaya, B. Saraçoğlu, İ. Kınac1, A Lifetime Regression Analysis with Unit Lindley-Weibull Distribution and it’s Application to Larynx Cancer Study, 2020. Under review, Advance Online publication.

12. C. Chesneau, K. Karakaya, H.S. Bakouch, C. Kus, An Alternative Approach to the Marshall-Olkin Family of Distributions: Weibull Baseline Case with Applications, 2020, Under review, Advance Online publication.

13. D.C.T. Granzotto, F. Louzada, The transmuted log-logistic distribution: modeling, inference, and an application to a polled tabapua race time up to first calving data, Commun. Stat. Theory Methods. 44 (2015), 3387-3402.

14. R.D. Gupta, D. Kundu, Aust. N. Z. J. Stat. 41 (1999), 173-188.

15. G.R. Aryal, C.P. Tsokos, Eur. J. Pure Appl. Math. 4 (2011), 89-102.

16. M.R. Mahmoud, R.M. Mandouh, J. Appl. Sci. Res. 9 (2013), 5553-5561.

17. J.F. Lawless, Statistical Models and Methods for Lifetime Data, vol. 362, JohnWiley and Sons, New York, NY, USA, 2011. 